aminés n au nitrogène total ; il nous est fourni par la valeur correșpondant au point B, dans notre cas $8,80 \%$.

4. Rapport du nitrogène protéique au nitrogène total ; le point $D$ nous la donne, dans notre cas $72,55 \%$.

5. Rapport du nitrogène ammoniacal au nitrogène soluble; pour l'obtenir, il faut utiliser le faisceau de traits qui croisent le graphique; lorsque le point $\mathrm{C}$ et le symétrique du $\mathrm{A}$ du troisième côté se trouvent sur la même ligne la valeur est $25 \%$; si c'est le C qui est sur une ligne plus haute que le symétrique du $\mathrm{A}$, la relation est de moins $25 \%$; et, dans le cas où c'est le symétrique du A qui se trouve sur une ligne plus haute que eelle passant par le C, la relation est de plus de $25 \%$.

6. Rapport de " nitrogène ammoniacal et nitrogène des acides aminés » au nitrogène soluble; on raisonne de la même façon que dans le cas précédent, mais au lieu du symétrique du point $\mathrm{A}$, il faut considérer le symétrique du point $B$.

Nous pensons que l'aspect de la courbe Q - 1 - 2 - 3 nous permettra de poursuivre notre travail, de connaître de nouvelles caractéristiques de la maturation du fromage et s'il en est ainsi, nous en rendrons compte dans une communication ultérieure.

(Laboratoires de Chimie de l'Institut Municipal d'Hygiène de Zaragoza (Espagne).)

\title{
LA SOLUBILISATION DE LA CASÉINE PAR L'AMIMONIAQUE : \\ SON UTILISATION DANS LA RECHERCHE \\ DES FALSIFICATIONS DU LAIT FRAIS PAR ADDITION DE LAIT EN POUDRE
}

\author{
par \\ GEORgES VALLIER
}

Notre étude porte sur les laits frais crus et sur des laits reconstitués à partir de la poudre de lait obtenue par le procédé Spray, utilisée dans la plupart des falsifications.

Cette poudre de lait est un lait complet d'origine hollandaise. Aucune indication de fabrication ne nous est connue, en particulier la température de préchauffage.

Principe. - Nous utilisons la solubilisation de la caséine du lait par l'ammoniaque.

Cette solubilisation est utilisée dans la méthode d'Adam MELLIÈRE, pour le dosage de la matière grasse du lait et dans la réaction 
d'UmiкоғF qui permet de déceler les falsifications du lait de femme par du lait de vache, c'est done une méthode connue.

Mais, à cette action de l'ammoniaque sur le lait, nous avons associé l'action de la température utilisée comme floculant des protéines autres que la caséine et l'action de l'alcool amylique, utilisée comme dissolvant des graisses.

Technique. - Nous opérons selon la technique suivante :

Dans un tube à essai, nous mesurons $5 \mathrm{~cm}^{3}$ de lait. Nous ajoutons $4 \mathrm{~cm}^{3}$ d'une solution d'ammoniaque du commeree, diluée à $50 \%$, puis $1 \mathrm{~cm}^{3}$ d'alcool amylique.

Après agitation, le tube est mis au bain-marie à la température de $77^{\circ} 5$ pendant 90 minutes.

Expériences. - $1^{\circ}$ Avec des laits crus, nous constatons la formation d'une couche de crème à la surface. Le liquide restant prend une belle teinte porto avec un léger trouble visible à la lumière du jour.

Le léger trouble qui se manifeste parait dû aux albumines et globulines qui ont floculé. A. Rochaix et A. TAPERnoux donnent pour temps nécessaire à la coagulation de ces protéines, 30 minutes à $80^{\circ}$ et une heure à $77^{\circ} 5$. La température de notre bain-marie oscille entre $75^{\circ}$ et $80^{\circ}$, mais $1 \mathrm{~h} .30$ est nettement suffisant cependant pour floculer toutes les albumines et globulines du lait.

La coloration porto, est dûe en grande partie à la caséine. Si nous ajoutons au lait ainsi traité du chlorure de sodium jusqu'à saturation, nous obtenons une coagulation de la caséine. Le coagulum est brunâtre et laisse exsuder un sérum de couleur jaune clair comparable à la couleur de l'ammoniaque en solution diluée. Le sérum conserve une partie du floculat de l'albumine et de la globuline visible au léger trouble qu'il forme.

Cette saturation par le chlorure de sodium montre d'autre part que la caséine était bien solubilisée et non détruite.

$2^{\circ}$ Avec des laits reconstitués. La réaction est toute différente.

Le lait prend une teinte café au lait et une couche de crème se forme à la surface. L'examen à la lumière du jour montre que le liquide est opaque.

La couche de crème est moins importante que dans le lait frais. Il reste une partie des globules graisseux dans le liquide. Ce fait s'explique par la division en très fines goutelettes de la matière grasse du lait en poudre (A. C. Villanova et O. Ballarin.)

Par centrifugation on peut éliminer la totalité de la matière grasse du lait.

Le liquide ainsi obtenu est stable. Après une semaine aucun dépôt ne se forme. Au microscope apparait comme une suspension 
de grosses micelles qui sont des particules de caséine colorées.

Si nous ajoutons du chlorure de sodium jusqu'à saturation, nous voyons se former un coagulum de caséine qui laisse exsuder, comme dans le cas du lait frais, un sérum jaune clair ayant un léger trouble.

Nous pouvons en déduire tout d'abord, étant donnée la température de la réaction et par le fait que le trouble d $\hat{u}$ aux albumines et globulines est visible après action du chlorure de sodium, que ces protéines sont déjà floculées dans la réaction de l'ammoniaque. D'après A. RocharX et A. TAPERNoUX, la saturation du lait par le chlorure de sodium ne permet pas de floculer l'albumine et la globuline, mais la caséine coagule. Nous pouvons penser que la caséine n'est pas détruite, mais que ses propriétés sont modifiées dans le lait reconstitué.

La première origine des modifications que l'on puisse envisager, est celle dûe à la chaleur mise en œuvre lors du préchauffage. L. J. Manus et U.S. Ashworth ont montré que la température avait une grande influence sur les propriétés des protéines du lait et sur la caséine en particulier. Mais ces modifications portent surtout sur la solubilité de la poudre dans l'eau. Nous avons eu à examiner des laits reconstitués avec des poudres de très grande solubilité puisque pratiquement, aucun dépôt ne se formait par centrifugation.

Sans exclure la possibilité d'action de la chaleur sur la solubilité de la caséine dans les solutions ammoniaeales, il semble que la modification de la caséine des laits en poudre, au cours du stockage, soit à envisager. K. M. HENRy et S. K. Kon montrent qu'il y a blocage des groupes aminés libres de la caséine par un sucre réducteur : le lactose, pendant le stockage.

A. C. Villanova et O. Ballarin étudiant la poudre de lait Spray au microscope électronique n'ont pas mis en évidence de cristaux de lactose dans la poudre. Or, il est facile, d'après PoloNovskr M. d'obtenir des cristaux de lactose à partir d'une solution aqueuse. Celà nous incite à penser que dans le lait en poudre toute la caséine subit cette action du lactose. Il en résulterait une modification du comportement de la caséine vis-à-vis de l'ammoniaque. C'est peut-être au complexe caséine lactose que l'ammoniaque s'unit pour donner ces grosses micelles visibles au microscope.

Ce qui est certain, c'est que la caséine n'est pas solubilisée. Elle donne au lait reconstitué une densité optique différente de celle des laits frais et uné couleur café au lait qui ne peut se comparer à la couleur porto des laits frais.

C'est sur cette différence de densité optique dûe à la caséine qu'est basée notre méthode de dosage du lait en poudre dans les laits falsifiés. 


\section{Dosage de la poudre de lait dans un lait falsifié}

$1^{0}$ Nous avons construit un appareil permettant d'effectuer de façon pratique des mesures de densité optique sur les tubes à essai servant aux réactions.

L'appareil comprend essentiellement une source lumineuse constituée par une lampe de 12 volts qui donne un faisceau de rayons parallèles. Un filtre est posé sur le trajet des rayons lumineux au niveau d'une fente verticale de $3 \mathrm{~mm}$. sur $25 \mathrm{~mm}$. pratiquée sur une des faces de l'appareil.

A l'intérieur de cet appareil, se trouve un groupe fixe avec une fente verticale de même dimension que l'ouverture précédente.

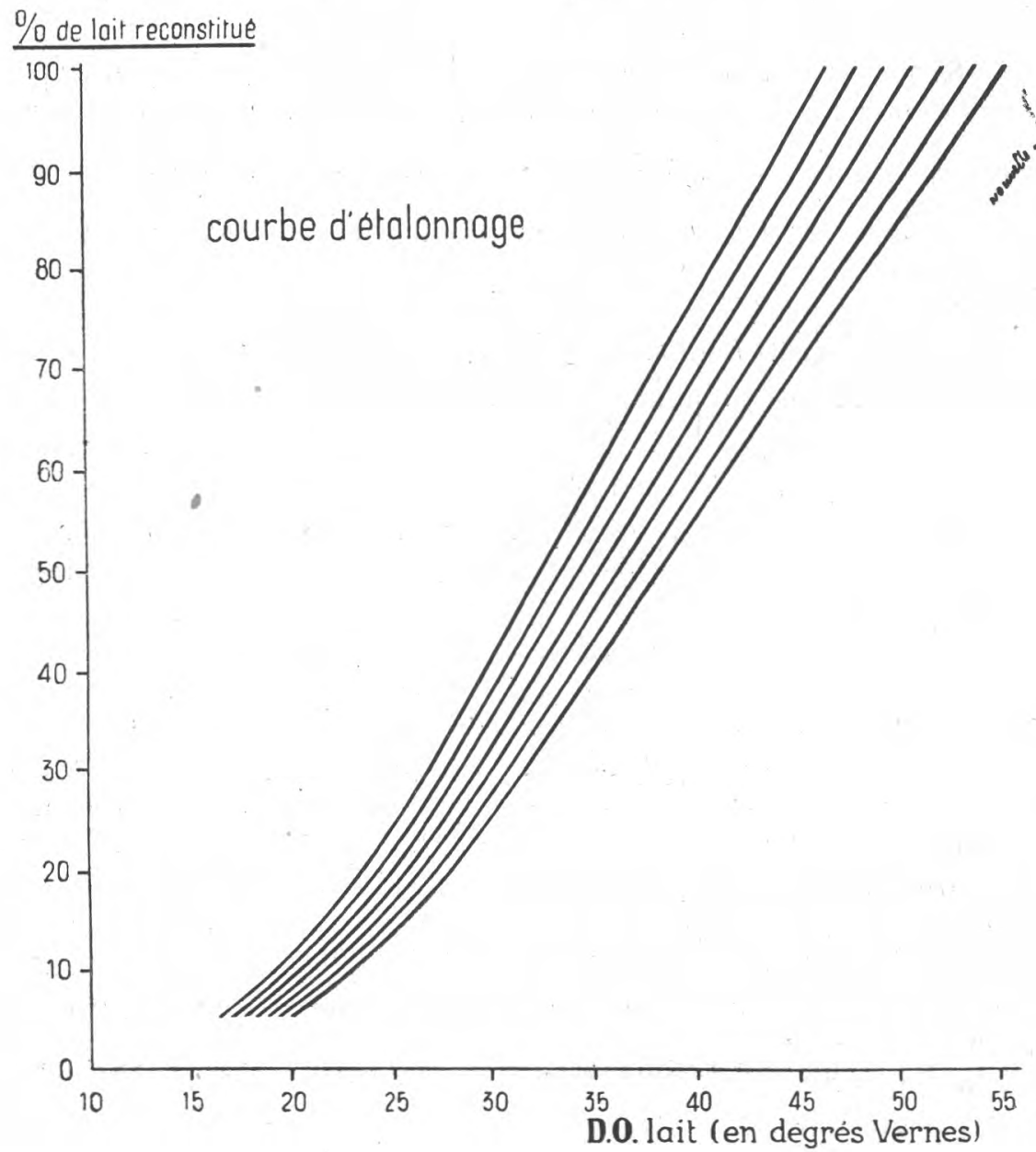


Un porte-tube permet de maintenir les tubes à essai contre l'ouverture verticale du groupe fixe et à une hauteur telle l'axe optique des rayons lumineux traverse le tube au milieu de la hauteur de liquide.

Une cellule photoélectrique est placée face à l'ouverture du groupe fixe dans l'axe des rayons lumineux.

L'appareil constitue une chambre noire. Une ouverture au niveau du cadran de la cellule permettant de lire les graduations.

$2^{\circ}$ Mesures. Nous avons utilisé des tubes de $13 \mathrm{~mm}$. de diamètre et de 1,4 mm. d'épaisseur.

Nos mesures sont effectuées sur des mélanges de concentration connue en poudre de lait. Les tubes à essai sont mis à refroidir après la réaction et la lecture se fait toujours à la même température.

Si nous connaissons la transmission des mélanges de lait traité, par l'ammoniaque, nous pourrons déterminer la densité optique par la formule : d.o (en degrés Vernes) $=100 \log \frac{\mathrm{I}}{\mathrm{T}}$. La transmission $\mathrm{T}$ est égale au rapport des intensités lumineuses incidentes et transmises $\frac{\mathrm{I}}{\mathrm{I}^{\circ}}$.

I est l'intensité lumineuse du faisceau transmis ;

Io est l'intensité lumineuse du faisceau incident.

$3^{\circ}$ Précision des mesures. La précision des mesures varie avec le pourcentage de lait reconstitué.

Elle est fonction d'autre part de la sensibilité de la cellule photoélectrique. Les graduations plus étalées permettent des mesures plus précises.

Une intensité du faisceau lumineux suffisamment importante a permis de placer au maximum de sensibilité de la cellule photoélectrique la mesure des faibles densités optiques correspondant aux laits les moins falsifiés.

Des caractéristiques des courbes d'étalonnage nous pouvons, déduire la précision des mesures.

Prenons la courbe des laits de densité égale à 1.034 pour un lait falsifié à $5 \%$, nous avons une transmission égale à $\frac{\mathrm{I}}{\mathrm{I}^{\circ}}=\frac{6,95}{10,5}$, les transmissions minima et maxima que l'on puisse apprécier sont : $\frac{6,9}{10,6}$ et $\frac{7}{10,4}$ qui correspondent à des densités optiques de 18,7 et 17,3 degré Vernes. 
La courbe $\mathrm{d}=1,034$ donne des pourcentages en lait reconstitués qui sont de $6 \%$ et de $4 \%$ pour ces densités optiques.

Nous avons donc une erreur qui sera au maximum de $2 \%$.

En pratique, les erreurs que nous avons eu étaient de l'ordre de $1,5 \%$.

Pour les laits falsifiés à $70 \%$ sur la courbe, nous avons une transmission égale à : $\frac{\mathrm{I}}{\mathrm{I}^{\mathrm{o}}}=\frac{4}{10,5}$.

Les transmissions minima et maxima que l'on puisse apprécier sont : $\frac{3,9}{10,6}$ et $\frac{4,1}{10,4}$ qui correspondent à des densités optiques de 43,5 et 40,5 degrés Vernes. Les pourcentages en lait reconstitué correspondants sont : $76 \%$ et $66 \%$.

Nous avons donc une erreur qui sera au maximum de $10 \%$, en pratique les erreurs que nous avons eu étaient de l'ordre de $7 \%$.

Une plus grande précision est obtenue en diluant les laits fortement falsifiés avec des laits frais, car nous opérons alors dans les graduations étalées de la cellule photoélectrique.

\section{CONCLUSION}

Nous pensons que la méthode est applicable avec suffisamment de précision dans la pratique.

Cette méthode permettra de considérer comme nettement falsifiés, des laits falsifiés avec plus de $5 \%$ de lait reconstitué.

(Service de l'Elevage du Maroc. - Laboratoire des Abattoirs municipaux. Fés.)

\section{BIBLIOGRAPHIE}

N. Fiessinger, H. R. Olivier et M. Herbain. Diagnosties biologiques et fonetionnels, un volume. Editeur : Maloine, Paris, 1949.

K. M. Henry et S. K. Kon. Détérioration pendant la conservation du lait écrémé. Journal of Dairy Research, 1948, 3, 292-363, cité par C. WoLF, dans Le Lait, 303-304, 186.

L. J. Manus et U. S. Ashworth. Rapport de la qualité de la conservation, de la solubilité et de la densité du lait entier en poudre avec quelques variations de la fabrication. II. Solubilité et densité. Journal of Dairy Science, 1948, 11, 935-945. Cité par C. Wolf, dans Le Lait, 1951, 305306, 281.

M. Polonoviki, P. Boulanger, M. Machebcuf, J. Roche et C. Sannie. Biochimie Médicale. Un volume. Editeur : Masson, Paris, 1948.

A. Rochaix et A. Tapernoux. Le lait et ses dérivés. Un volume. Editeur : Vigot Frères, Paris, et Librairie Médicale, Lyon, 1948.

A. C. Villanova et O. Ballarin. Etude au microscope électronique de la structure du lait en poudre Spray. Le Lait, 1950, 293-194, 119 et 122. 


\title{
OBSERVATIONS SYNTHÉTIQUES ET MESURES A Conseiller en VUE de préparer unE CASÉINE LACTIQUe de LA MEILLEURE QUALITÉ (1)
}

\author{
par
}

\section{Agusto A. SCHNACK}

La qualité fait le prix. Ceci n'est pas nouveau dans notre marché de caséine. Il est cependant possible que les acheteurs habituels de ce produit soient obligés de rectifier leur jugement en se servant de données plus rigoureuses pour en apprécier les qualités, ceci en raison des récentes dispositions prises par les organismes respectifs de l'Etat, chargés de rechercher les améliorations techniques et économiques de la production et du commerce, ces mesures devant être présentement déjà en vigueur.

Comme conséquence immédiate de ces mesures, quelques producteurs de caséine se sont trouvés en face de plus grandes exigences pour obtenir des cotations meilleures dans la vente de leur production. Ceci va les mettre dans l'obligation de réviser leurs méthodes de travail, de les réformer si cela est nécessaire et dans certains cas, d'abandonner de vieux systèmes de production pour d'autres plus en rapport avec les conditions actuelles.

En termes généraux, il convient de rappeler qu'une caséine lactique s'obtient en la séparant, avec un haut degré de pureté, des autres éléments du lait écrémé; elle est donc pour ainsi dire pratiquement pure ou avec un pourcentage très bas de matière grasse, de lactose et de sels minéraux et sa teneur en eau est réduite au minimum. Sa couleur doit aller du blane jusqu'à un ton légèrement crémeux et son arome doit rappeler celui du lait frais. La saveur doit être douce, sans acidité.

La Direction Laitière du Ministère de l'Agriculture de la République Argentine applique pour la caséine lactique, les normes suivantes, aux facteurs physiques et chimiques, correspondant à la qualité "Extra»:

Solubilité: complète (on admet quelques points blancs); Couleur: blanc cristallin; Odeur: naturelle; Consistance: Bonne (assez fluide); Aspect: propre (rigoureusement); Acidité totale: jusqu'à 9,5 (pas moins de 7,5 $\left.\mathrm{cm}^{3}\right)$; Graisse: maximum $1 \%$; Cendres: maximum $3 \%$.

La solubilité, la couleur, l'odeur, la consistance et l'aspect sont déterminés en $15 \%$ de borax, à quoi l'on ajoute $40 \mathrm{~cm}^{3}$ d'eau

(1) La Industria Lechera, 1954, 418, 239 (traduction de Mlle C. Sellet). 